\title{
Effects of anticancer agents on cell viability, proliferative activity and cytokine production of peripheral blood mononuclear cells
}

\author{
Hiromi Sakai, ${ }^{1}$ Satoshi Kokura, ${ }^{1,2, *}$ Takeshi Ishikawa, ${ }^{1,2}$ Reiko Tsuchiya, ${ }^{1}$ Manabu Okajima, ${ }^{1}$ \\ Tatsuzou Matsuyama, ${ }^{1}$ Satoko Adachi, ${ }^{2}$ Kazuhiro Katada, ${ }^{1}$ Kazuhiro Kamada, ${ }^{1}$ Kazuhiko Uchiyama, ${ }^{1}$ \\ Osamu Handa, ${ }^{1}$ Tomohisa Takagi, ${ }^{1}$ Nobuaki Yagi, ${ }^{1}$ Yuji Naito' and Toshikazu Yoshikawa \\ 'Department of Gastroenterology and Hepatology and 2Department of Cancer ImmunoCell Regulation, Kyoto Prefectural University of Medicine, \\ 465 Kajii-cho, Kamigyo-ku, Kyoto 602-8566, Japan
}

\begin{abstract}
We investigated the effects of anticancer agents on peripheral blood mononuclear cells for the purpose of providing data to support new translational chemoimmunotherapy regimens. Peripheral-blood mononuclear cells were treated with one of four anticancer agents (5-fluorouracil, irinotecan, cisplatin, and gemcitabine) for $\mathbf{2} \mathrm{h}$, after which cell viability was determined. For assessment of effects of each drug on proliferation and cytokine production, cells were stimulated with phytohemagglutinin for $48 \mathrm{~h}$. As a result, the anticancer agents did not affect cell viability. Cell proliferation was unaffected by 5 -fluorouracil and irinotecan but inhibited by cisplatin and gemcitabine. Treatment with gemcitabine enhanced the production of IFN- $\gamma$ and decreased the number of regulatory $T$ cells. gemcitabine treatment increased IFN- $\gamma$ production among CD4 $\mathrm{T}$ cells but not among CD8 $\mathrm{T}$ cells. The results indicated that GEM had immunoregulatory properties that might support immune response against cancer. This finding has implications for designing chemoimmunotherapy strategies.
\end{abstract}

Key Words: chemoimmunotherapy, 5-fluorouracil (5-FU), irinotecan (CPT-11), cisplatin (CDDP), gemcitabine (GEM)

\footnotetext{
Cytotoxic chemotherapy, surgery, and radiotherapy are the major modalities used in the treatment of human malignancy. Unfortunately, both chemotherapy and radiotherapy are often limited by toxicities in normal tissues, and the effects of these conventional therapies may not be sufficient in advanced malignancies. Strategies that kill cancer cells efficiently by using the correct combination and schedule of conventional therapies, but that also stimulate the host immune system might aid in tumor regression. Combinations of chemotherapy and immunotherapy could potentially act synergistically against malignancies.

Cytotoxic chemotherapy is generally regarded as immunosuppressive because of its effects on dividing cells in bone marrow and peripheral lymphoid tissue. However, accumulating evidence indicates that cytotoxic anticancer agents also affect the immune system to contribute to tumor regression. Several anticancer agents have been reported to upregulate cell surface expression of major histocompatability complex (MHC) molecules. ${ }^{(1)}$ The agents 5-fluorouracil (5-FU), irinotecan (CPT-11) and cisplatin (CDDP) have been shown to enhance ICAM-1 and Fas expression, increasing sensitivity to lysis by cytotoxic $\mathrm{T}$ lymphocytes (CTLs). ${ }^{(2)}$ Gemcitabine (GEM) has been reported to have multiple

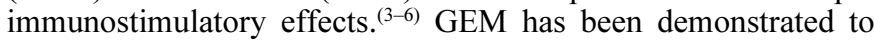
reduce myeloid-derived suppressor cell (MDSC) levels, reducing interleukin (IL)-10 levels and restoring macrophage production of IL-12.(4)
}

These reports suggest the possibility that combination of chemotherapy with immune therapy might produce therapeutic synergy through the immunomodulatory effects of the anticancer agents. However, effects of anticancer agents on immune cells are not entirely clear. In this study, we tested the effects of anticancer agents on peripheral-blood mononuclear cells (PBMCs) for the purpose of providing data to support the design of new translational regimens combining chemotherapy and immunotherapy.

\section{Materials and Methods}

Blood sampling and isolation of PBMCs. Blood samples were obtained from at least three different healthy volunteers who gave informed consent for participation in the study. Twenty milliliters of venous blood was collected in heparinized tubes. After centrifuging, plasma was removed. The layer that included peripheral blood mononuclear cells was gathered with a pipette and diluted in RPM1-1640 (Wako Pure Chem. Ind. Ltd., Osaka, Japan). Ficoll-Paque ${ }^{\mathrm{TM}}$ (GE Healthcare Bioscience AB, Uppsala, Sweden) was added and blood cells were isolated. PBMCs were localized in the second creamy layer from the top. They were mixed with Turk's solution for leukocyte counting (MERCK, Tokyo, Japan) in a 96-well plate. Cells were counted in an erythrocytometer and cell concentrations were calculated.

Anticancer drugs. The four drugs tested were: 5-FU (Kyowa Hakko Kirin, Tokyo, Japan), CPT-11 (Yakult, Tokyo, Japan), CDDP (Nippon Kayaku, Tokyo, Japan), and GEM (Eli Lilly, Tokyo, Japan). Phytohemagglutinin-L (PHA) was purchased from Roche Applied Science in Germany.

Assessment of cell viability. The effects of each anticancer agent on viability of PBMCs were determined by WST- 8 assay (Dojin Laboratory, Kumamoto, Japan) according to the manufacturer's instructions. PBMCs and incremental concentrations of each anticancer agent were incubated at $37^{\circ} \mathrm{C}$ for $2 \mathrm{~h}$ in tilted centrifuge tubes with loosened caps. The concentrations of each anticancer agent in our experiment were based on plasma levels of each drug in clinical use in monotherapy. We referred to the time change of the blood level of each anticancer agent to decide the stimulation time for it. After stimulation with anticancer agents for $2 \mathrm{~h}$, cells were washed with RPMI 1640 containing 5\% fetal calf serum (FCS), and the stimulation was stopped. PBMCs were resuspended at a concentration of $1 \times 10^{5}$ cells/well in $100 \mu \mathrm{l}$ of WST-8 solution and seeded into a $96-$ well plate, where they were

*To whom correspondence should be addressed. E-mail: s-kokura@koto.kpu-m.ac.jp 
incubated at $37^{\circ} \mathrm{C}$ for $2 \mathrm{~h}$. Absorbance of each well was measured at dual wavelengths of $450 / 600 \mathrm{~nm}$ using a microplate reader (MPR-A4i, Tosoh Corporation, Tokyo, Japan).

PBMC proliferation assay. The bromodeoxyuridine (BrdU) cell proliferation assay (Chemicon International, Temecula, CA) was used to detect cell proliferation. After stimulation with incremental concentrations for $2 \mathrm{~h}$, PBMCs were washed with RPMI1640 to remove anticancer agents. PBMCs were then stimulated with PHA for $48 \mathrm{~h}(5 \mu \mathrm{g} / \mathrm{ml})$ and subsequently dispensed into a 96-well plate at a density of $1 \times 10^{5}$ cells/well. PBMCs were allowed to proliferate in growth conditions for $24 \mathrm{~h}$, pulsed with $20 \mu \mathrm{l} /$ well of BrdU solution, and incubated for $24 \mathrm{~h}$. After incubation, samples were processed according to the manufacturer's protocol for the BrdU cell proliferation assay. Absorbance of each well was measured at dual wavelengths of $450 \mathrm{~nm} / 600 \mathrm{~nm}$ using a microplate reader.

Cytokine assay. Simultaneous determination of levels of five cytokines was performed in PBMC culture supernatants. The cytokines measured were tumor necrosis factor- $\alpha$ (TNF- $\alpha$ ), interleukin-2 (IL-2), interleukin-4 (IL-4), interleukin-10 (IL-10), and interferon- $\gamma($ IFN- $\gamma)$. PBMCs were stimulated with incremental concentrations of anticancer agents for $2 \mathrm{~h}$. After stimulation, anticancer agents were washed out from PBMCs with RPMI-1640. PBMCs were then stimulated with PHA $(5 \mu \mathrm{g} / \mathrm{ml})$ and subsequently dispersed at a concentration of $2 \times 10^{5}$ cells/ well $/ 200 \mu$ into a 96 -well plate and incubated for $48 \mathrm{~h}$. Cytokine concentrations in PBMC culture supernatant were determined by cytokine-specific solid phase sandwich enzyme-linked immuosorbent assay (ELISA) kits (eBioscience, San Diego, CA and R\&D systems, Minneapolis, MN) according to the manufacturer's instructions.

Flow cytometric analysis. PBMCs were incubated with $10 \mu \mathrm{M}$ GEM for $2 \mathrm{~h}$ prior to stimulation with PHA $(5 \mu \mathrm{g} / \mathrm{ml})$. After $48 \mathrm{~h}$ of stimulation, expression of CD4 and CD8 leukocytes and intracellular staining for Foxp3 and IFN- $\gamma$ were measured with anti-CD4, anti-CD8, anti-Foxp3 and anti-IFN- $\gamma$ antibodies (eBioscience, San Diego, CA). Five hours before the cells were harvested, BrefeldinA (BD Biosciences, San Jose, CA) was added for intracellular blocking of IFN- $\gamma$. A single aliquot was thawed, and then mononuclear cells were stained with fluorescenceconjugated antibodies and analyzed with a FacsCalibur flow cytometer (BD Biosciences, San Jose, CA). The gating strategies for lymphocytes were based upon forward light scatter (size) and $90^{\circ}$ side scatter (granularity) as performed with these samples. Mouse immunoglobulin G1 (IgG1) conjugated with fluorescein isothiocyanate (FITC) or R-phycoerythrin (PE) served as negative controls. Harvested cells were processed according to the manufacturer's protocol for staining of surface cell markers and intracellular staining of mononuclear cells. PE-conjugated antibodies directed against IFN- $\gamma$ were used to identify $\mathrm{T}$ helper type 1 (Th1) cells. PE-conjugated antibodies directed against Foxp 3 were used to identify regulatory T cells. IFN $-\gamma$ staining was combined with FITC-conjugated anti-CD4 staining and allophycocyanin-conjugated anti-CD8 staining. Foxp3 staining was combined with FITC-conjugated anti-CD4 staining. Off-line analysis was performed using CELLQUEST software version 6.0 for MAC OS 10 (BD Biosciences, San Jose, CA).

Statistical analysis. ANOVA with post hoc testing was used for multiple comparisons. Differences were considered significant when $p<0.05$. Statistical analysis was conducted using StatView 5.0 for Windows (SAS Institute Inc., Cary, NC).

\section{Results}

Effects of anticancer agents on cell viability of PBMCs. Two-hour stimulation with the agents studied did not affect the viability of PBMCs measured by WST- 8 assay at each concentration (Fig. 1). There was also no effect on viability of PBMCs

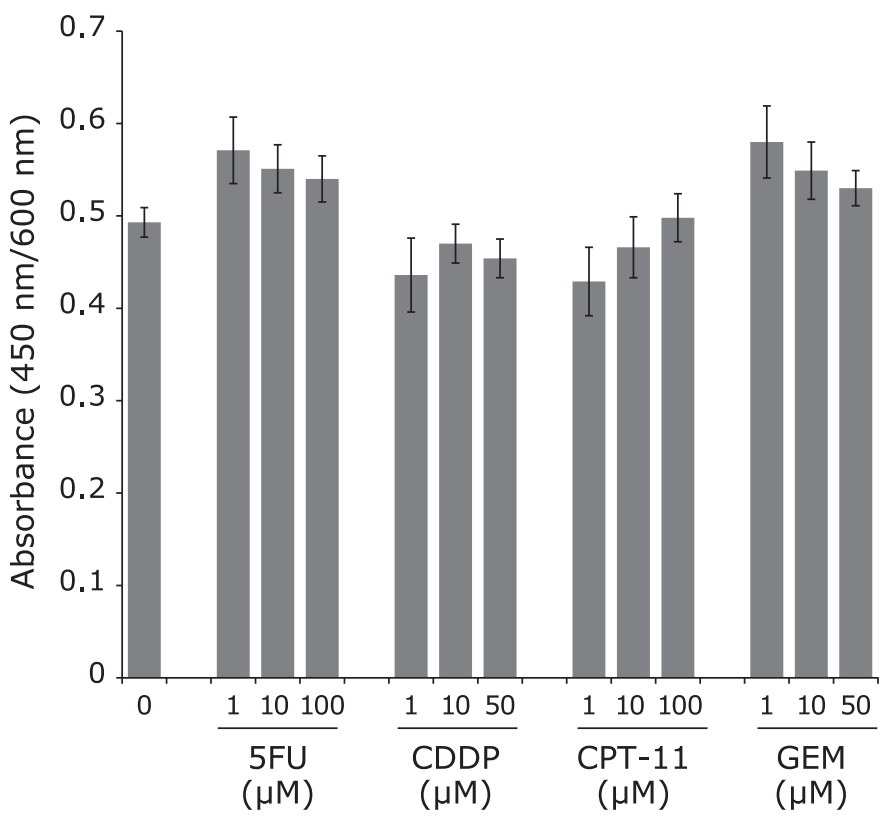

Fig. 1. Effects of anticancer agents on cell viability of PBMCs. PBMCs and incremental concentrations of each anticancer agent were incubated at $37^{\circ} \mathrm{C}$ for $2 \mathrm{~h}$. After stimulation of anticancer agents, cell viability of PBMCs was determined by WST-8 assay. Data are expressed as the mean \pm standard error of the mean (SEM).

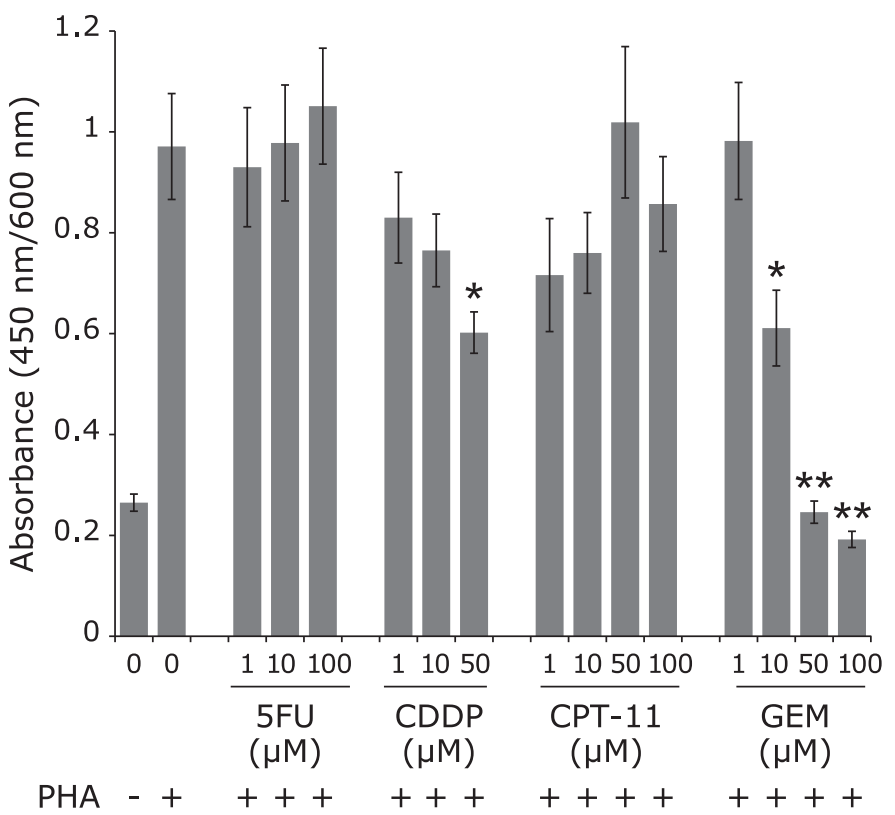

Fig. 2. Effects of anticancer agents on proliferative activity of PBMCs. After stimulation with incremental concentrations of anticancer agents for $2 \mathrm{~h}$, PBMCs were then stimulated with PHA $(5 \mu \mathrm{g} / \mathrm{ml})$ for $48 \mathrm{~h}$. Cells were pulsed with bromodeoxyuridine (BrdU) solution and measured using a microplate reader. Data are expressed as the mean $\pm \mathrm{SEM}$. ${ }^{*} p<0.05,{ }^{*} p<0.0001$, vs $\mathrm{PHA}(+)$, no anticancer agent.

based on Trypan blue staining (data not shown).

Effects of anticancer agents on proliferative activity of PBMCs. Neither 5-FU nor CPT-11 affected the proliferation potency of PBMCs stimulated with PHA (Fig. 2). Treatment with CDDP inhibited proliferation potency significantly at $50 \mu \mathrm{M}$ 
a
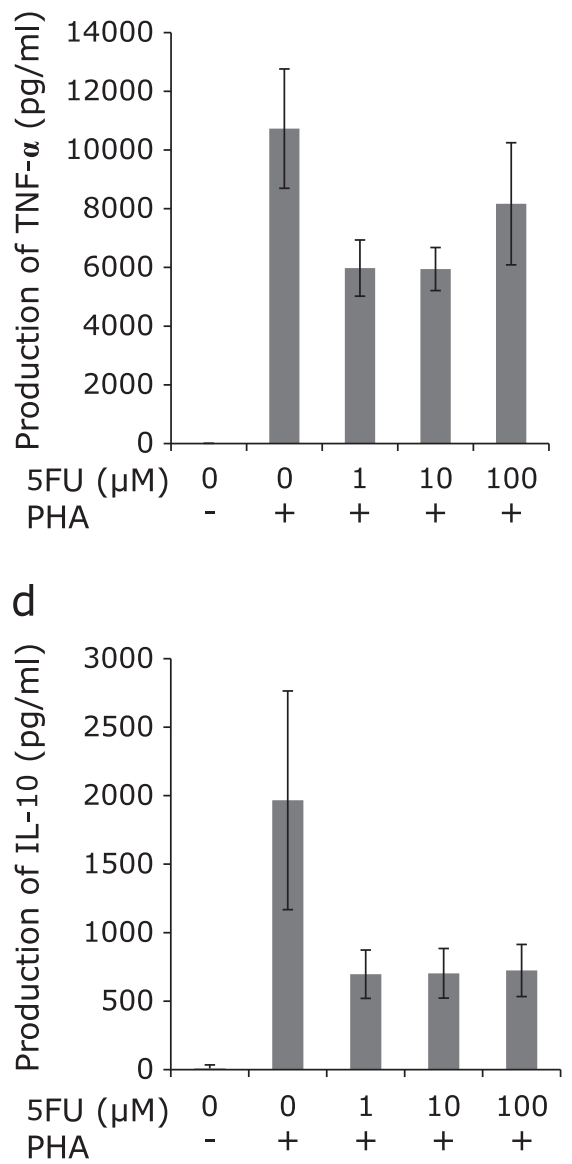

b
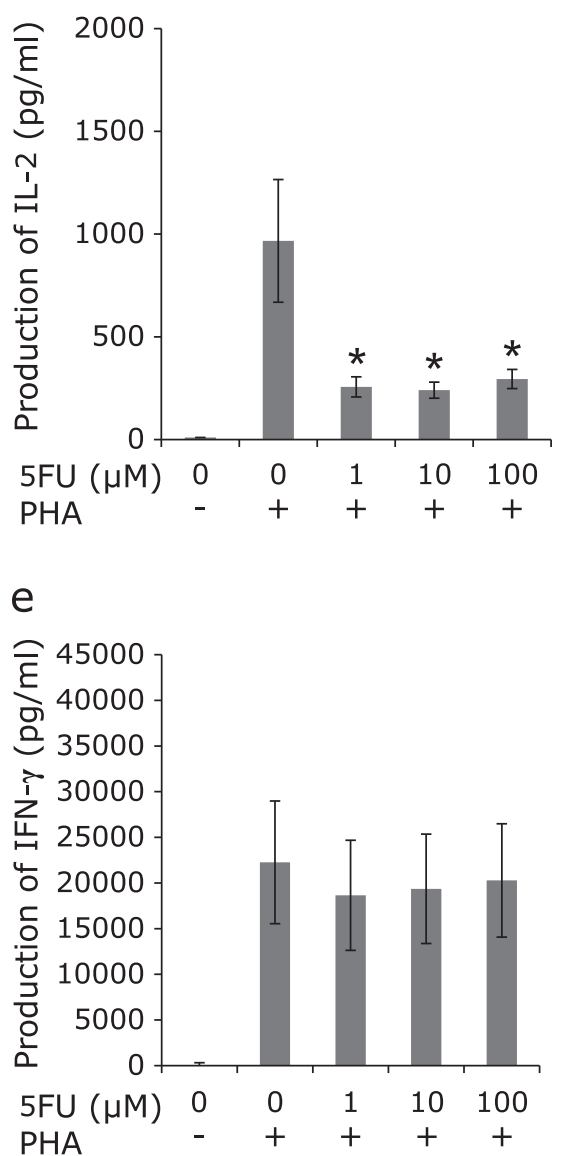

C

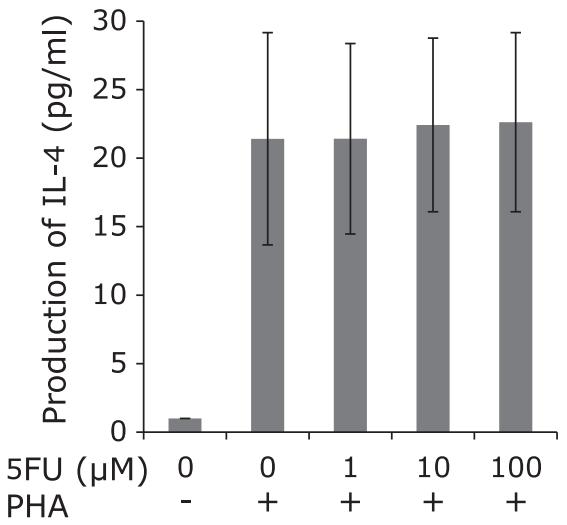

Fig. 3. Effects of 5-FU on PHA-induced cytokine production. After $2 \mathrm{~h}$ chemical stimulation with 5-FU, PBMCs were then incubated with PHA for $48 \mathrm{~h}$. Cell culture supernatant was assayed for TNF- $\alpha(\mathrm{a}), \mathrm{IL}-2(\mathrm{~b}), \mathrm{IL}-4(\mathrm{c}), \mathrm{IL}-10(\mathrm{~d})$ and IFN- $\gamma(\mathrm{e})$ by using a cytokine-specific solid phase sandwich ELISA. In all panels, data are expressed as the mean \pm SEM. * $p<0.05$, vs $\mathrm{PHA}(+)$, no anticancer agent.

$(p=0.0039)$. Treatment with GEM inhibited proliferation potency significantly at each concentration and caused a concentrationdependent inhibition. Thus, it is likely that GEM has a more profound suppressive effect on PBMC proliferation when compared with CDDP

Effects of anticancer agents on PHA-induced cytokine production. Treatment with 5 -FU inhibited the production of IL-2 significantly $(p<0.05)$ compared to stimulation with PHA alone, and also inhibited that of IL-10, but the effect did not reach significance $(p=0.0512)$ (Fig. $3 \mathrm{a}-\mathrm{e})$. The production of other cytokines (i.e., TNF- $\alpha$, IL- 4 and IFN- $\gamma$ ) was not affected by treatment with 5-FU. Results with CDDP were similar to those with 5-FU (Fig. 4 a-e). Treatment with CDDP significantly inhibited the production of IL-2 $(p<0.05)$, and also inhibited that of IL-10, but significance was not reached $(p=0.1651)$. The production of other cytokines (i.e., TNF- $\alpha$, IL-4 and IFN- $\gamma$ ) was not affected. Treatment with CPT-11 at concentrations of 10,50 and $100 \mu \mathrm{M}$ significantly inhibited the production of TNF- $\alpha$ $(p<0.05)$, and also significantly inhibited that of IL-2 at each concentration level $(p<0.05)$ (Fig. 5 a-e). Production of IL-4 was not affected, while that of IFN- $\gamma$ and IL-10 was inhibited, but not significantly ( $p=0.1086$ and $p=0.4584$, respectively). A significant increase in IFN- $\gamma$ production occurred only in PBMCs exposed to GEM at a concentration of $10 \mu \mathrm{M}(p=0.0188$, Fig. 6 $\mathrm{a}-\mathrm{e})$. Unlike other anticancer agents, the production of IL-2 was not inhibited by treatment with GEM. The production of TNF- $\alpha$ was significantly inhibited at 50 and $100 \mu \mathrm{M}(p<0.05)$. Production of IL-4 was not affected, and that of IL-10 was inhibited in a concentration-dependent manner without a significant difference.

Effects of GEM on lymphocyte populations and frequency of cells producing IFN- $\gamma$. We observed that treatment with GEM at a concentration of $10 \mu \mathrm{M}$ enhanced IFN- $\gamma$ production in PHA-stimulated PBMCs. We performed further imunocytofluorometric analysis to evaluate the effects of GEM on lymphocyte type and frequency of IFN- $\gamma$-producing cells. The percentage of CD4 and CD8 T-cell subsets of lymphocytes were not affected by treatment with GEM (Fig. 7 and 8). However, the percentage of regulatory $\mathrm{T}$ cells, defined as Foxp3-positive CD4 cells was significantly lower following exposure to GEM $(p=0.0269)$.

Intracellular staining studies revealed that there were few IFN$\gamma$-producing cells in PBMC cultures without PHA stimulation. With PHA stimulation, the frequency of IFN- $\gamma$-producing cells among both CD4 and CD8 T cells increased significantly. Treatment with GEM significantly increased the frequency of IFN- $\gamma$ producing CD4 cells $(p=0.0021)$ (Fig. 9 and 10). The frequency of IFN- $\gamma$-producing CD8 cells was unaffected by exposure to GEM.

\section{Discussion}

Various immunotherapies, such as vaccines directed against tumor-associated antigens and adoptive cell transfer, are currently being tested in clinical trials. However, there have been few studies combining immunotherapy and chemotherapy in cancer, 
a
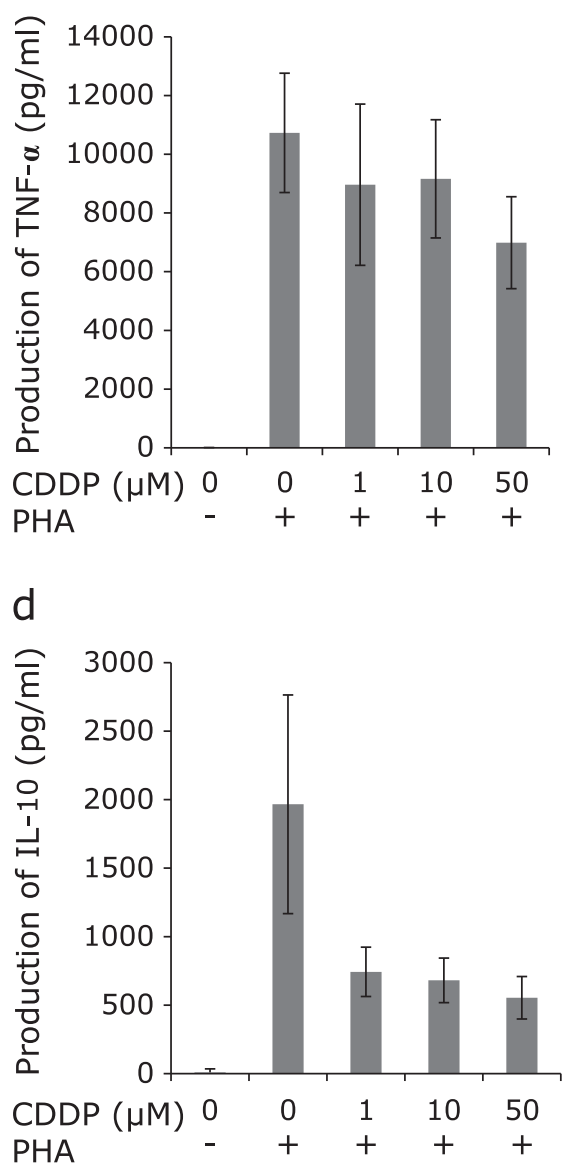

b
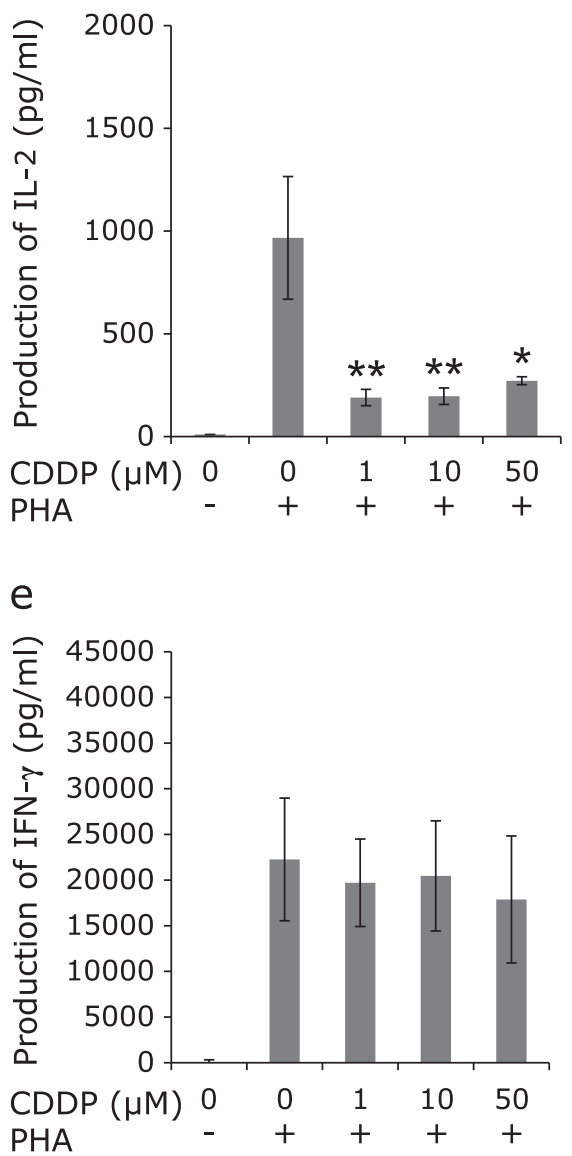

C

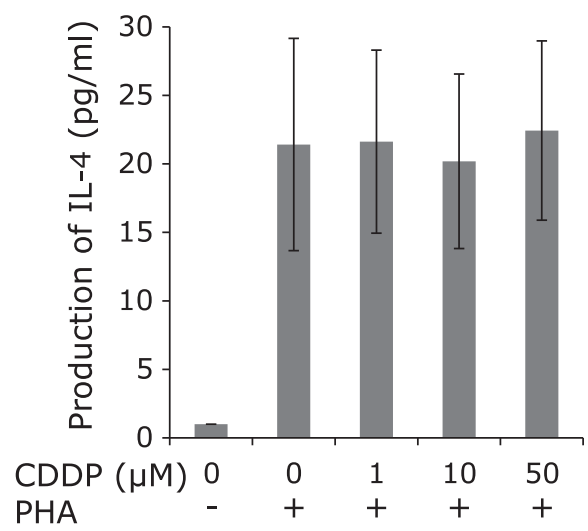

Fig. 4. Effects of CDDP on PHA-induced cytokine production. After $2 \mathrm{~h}$ chemical stimulation with CDDP, PBMCs were then incubated with PHA for $48 \mathrm{~h}$. Cell culture supernatant was assayed for TNF- $\alpha(\mathrm{a}), \mathrm{IL}-2(\mathrm{~b}), \mathrm{IL}-4(\mathrm{c}), \mathrm{IL}-10(\mathrm{~d})$ and IFN- $\gamma(\mathrm{e})$ by using a cytokine-specific solid phase sandwich ELISA. In all panels, data are expressed as the mean \pm SEM. ${ }^{*} p<0.05,{ }^{*} p<0.0001$, vs PHA(+), no anticancer agent.

mostly because it has been widely assumed that chemotherapy is immunosuppressive and would negate the potential benefits of immunotherapy. Recent evidence indicates that cytotoxic anticancer agents also affect the immune system, contributing to tumor regression. ${ }^{(1,7-9)}$ In this study, we examined the effects of anticancer agents on PBMCs to provide a foundation for a rational approach to chemotherapy that would enable the effective use of adjunctive immunotherapy.

We chose 5-FU, CDDP, CPT-11 and GEM for this study because they act on cancer cells through different mechanisms and are commonly used alone or in different combinations in the treatment of very common malignancies such as colorectal, pancreatic, gastric and breast cancers, as well as non-small cell lung cancer. In our study, none of these anticancer agents affected the viability of PBMCs. Contrary to our results, Alvino et al. ${ }^{(10)}$ reported that GEM inhibited the generation of lymphokineactivated killer cells and CTLs in different in vitro models in which PBMCs were treated with graded concentrations of GEM for $16 \mathrm{~h}$. In our study, PBMCs were treated with GEM for $2 \mathrm{~h}$, and the difference in exposure time might cause the differences in results of these studies. Plasma levels of GEM in clinical use decrease to $1 \%$ or less within $2 \mathrm{~h}$ of administration. Therefore, we believed it to be reasonable to stimulate PBMCs with GEM for $2 \mathrm{~h}$ in order to parallel clinical use.

Proliferative activity was analyzed using BrdU proliferation assay. Treatment with CDDP inhibited proliferation potency significantly at $50 \mu \mathrm{M}$ and did not affect it at lower concentrations. GEM inhibited proliferative activity significantly, in a concentration-dependent fashion. In agreement with our results, previous studies have demonstrated that GEM inhibits mitogeninduced proliferative activity of lymphocytes..$^{(11-13)}$ Nowak et al..$^{(13)}$ demonstrated that lymphocytes from GEM-treated mice exhibited decreased proliferative activity to both polyclonal T-cell mitogens (anti-CD3) and polyclonal B-cell mitogens (anti-IgM). However, in their mouse model, antigen-specific proliferative activity was augmented significantly. Clarification of the effect of GEM on proliferative activity of tumor antigen-specific lymphocytes in humans, especially in cancer patients, is needed.

Various cytokines produced by immune cells serve important roles in tumor immunity. ${ }^{(14)}$ In this study, we examined IL-2, IFN- $\gamma$, TNF- $\alpha$ (as a Th1 cytokine), and IL-4 and IL-10 (as a Th2 cytokine). Cytokine assay revealed that cytokine production profile associated with exposure to GEM was distinctly different from those observed with the other three anticancer agents. IL-2 is produced by naïve $\mathrm{T}$ cells and helper $\mathrm{T}$ cells and acts to activate CTLs and natural killer cells. ${ }^{(15)}$ In this study, 5-FU, CDDP and CPT-11, but not GEM, significantly inhibited the production of IL-2. As for IFN- $\gamma$, GEM at a concentration of $10 \mu \mathrm{M}$ significantly enhanced the production of IFN- $\gamma$. However, it is unclear why there was no concentration-dependent increase of IFN- $\gamma$ production with higher concentrations of GEM. The inhibitory effects of GEM on PBMC proliferation, which occurs in a concentration- 
a
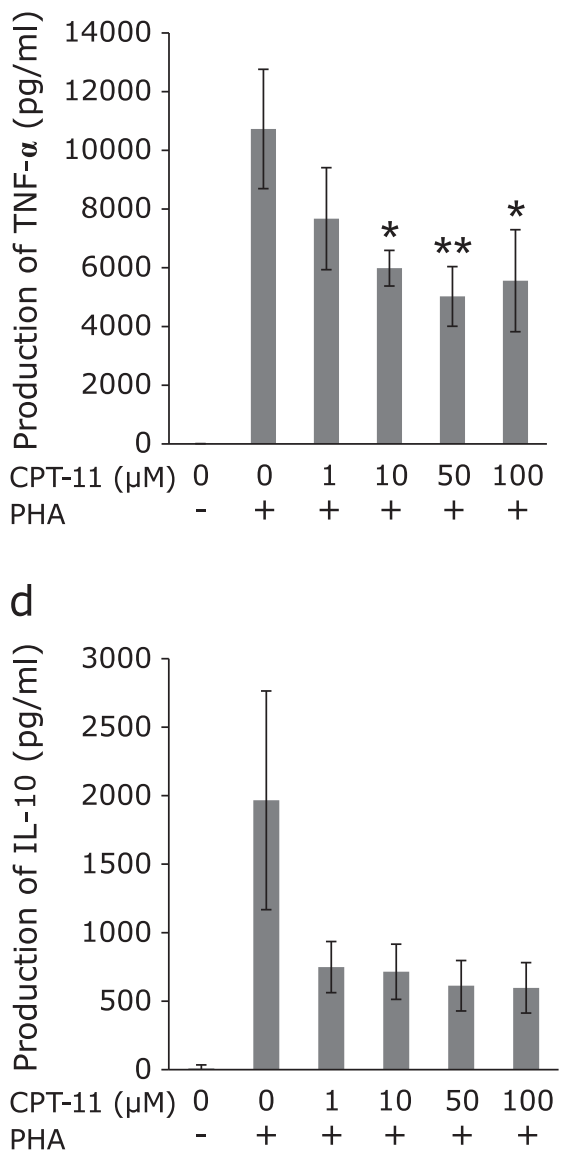

b
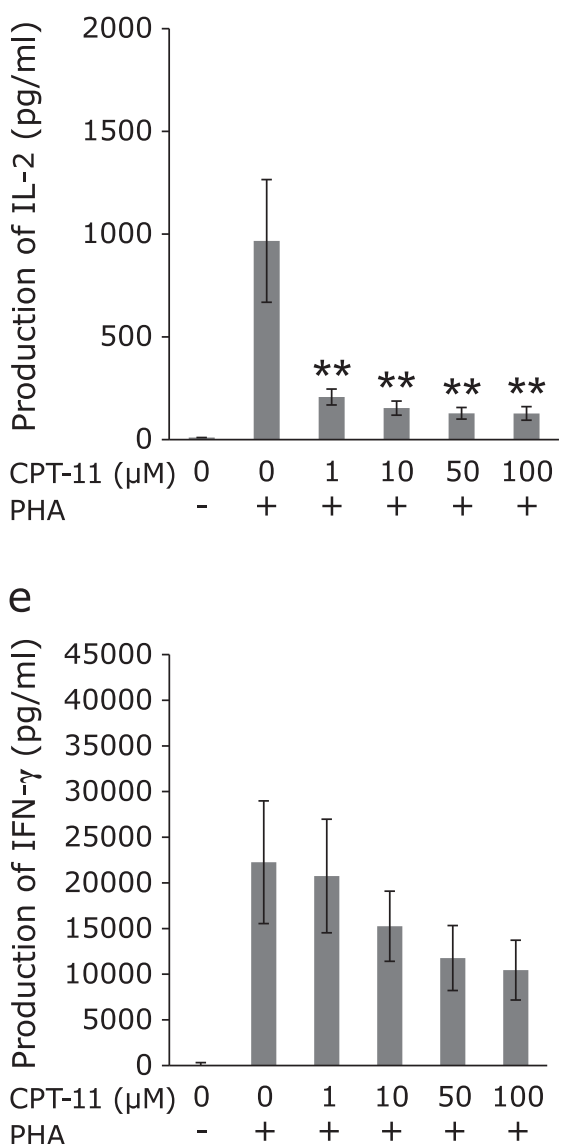

C

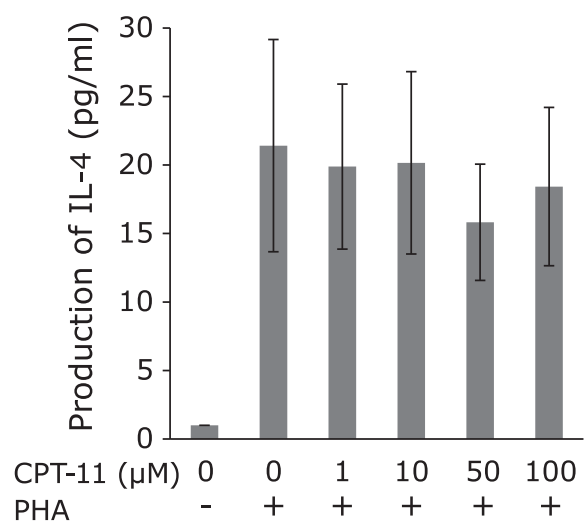

Fig. 5. Effects of CPT-11 on PHA-induced cytokine production. After $2 \mathrm{~h}$ chemical stimulation with CPT-11, PBMCs were then incubated with PHA for $48 \mathrm{~h}$. Cell culture supernatant was assayed for TNF- $\alpha(\mathrm{a}), \mathrm{IL}-2(\mathrm{~b}), \mathrm{IL}-4(\mathrm{c}), \mathrm{IL}-10$ (d) and IFN- $\gamma(\mathrm{e})$ by using a cytokine-specific solid phase sandwich ELISA. In all panels, data are expressed as the mean \pm SEM. ${ }^{*} p<0.05,{ }^{*}{ }^{*} p<0.0001$, vs $\mathrm{PHA}(+)$, no anticancer agent.

dependent manner, might help to explain this, in part. Previous studies have shown that GEM could enhance IFN- $\gamma$ production from immune cells. ${ }^{(16)}$ Plate et al. ${ }^{(17)}$ demonstrated that the number of IFN- $\gamma$-producting $\mathrm{T}$ cells increased in most patients after treatment with GEM. Recent reports indicate that GEM reduces the number of myeloid-derived suppressor cells (MDSCs), which are considered to play an important role in tumor-associated immune suppression, and hence GEM was theorized to promote the activities of CD8 $\mathrm{T}$ cells ${ }^{(18)}$ and increase the production of IFN- $\gamma$. With regard to Th2 cytokines, the four anticancer agents examined in this study showed a tendency to inhibit the production of IL-10 but did not have an effect on the production of IL-4. Sinha et $a{ }^{(4)}$ also reported that GEM inhibited the production of IL-10 by inhibition of MDSCs and promoted the production of IL-12 from macrophages. Thus, GEM could boost tumor immune reactivity by enhancing production of IFN $-\gamma$ and shifting cytokine balance to Th1.

Next, we examined the effects of GEM on lymphocyte type and the frequency of IFN- $\gamma$-producing cells. Treatment with GEM increased the frequency of IFN- $\gamma$-producing cells among CD4 T cells, while frequency among CD8 T cells was not affected. These results suggest that the main source of effects on IFN- $\gamma$ from GEM is mediated by CD4 T cells. Also noteworthy is our finding that treatment with GEM significantly decreased the percentage of Tregs. Treg is one of the most potent and well-studied suppressive cell types found in the tumor microenvironment. ${ }^{(19)}$ Previous studies have demonstrated that removal of Tregs results in the enhancement of effective tumor immune responses via the removal of strong immunosuppression, ${ }^{(20-24)}$ and clinical studies have demonstrated that Treg depletion alone or in combination with active immunotherapy increased effector $T$ cell activation. ${ }^{(21,22)}$ In this context, the characteristics of GEM, including ability to enhance IFN- $\gamma$ production and to downregulate Tregs, are expected to act synergistically in combination with immunotherapy against cancer. Kimura et $a l .{ }^{(25)}$ have actually demonstrated that GEM could enhance therapeutic effects of immunotherapy in patients with pancreatic cancer. In this study, patients receiving dendritic cell (DC) vaccine combined with GEM had a superior overall survival compared to those receiving DC vaccine combined with $\mathrm{S}-1$, an oral fluoropyrimidine.

There are several limitations associated with our study. We tested PBMCs from healthy donors but not from cancer patients. In addition, lymphocytes were activated by PHA, but not by cancer-specific antigens. These factors might limit the applicability of our results to cancer cells and patients. Although we evaluated the effect of each individual anticancer agent on PBMCs in this study, combination therapy of anticancer agents is the mainstream treatment for many types of cancer in clinical setting. Additional study is required for understanding immunemodulating effects of the combination of anticancer agents on PBMCs.

In conclusion, none of four anticancer agents affected the viability of PBMCs. GEM had unique immunoregulatory properties that might promote an immune response against cancer cells. The 
a

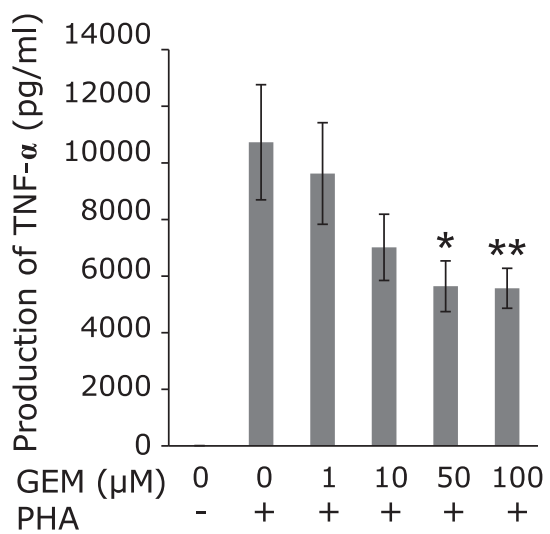

d

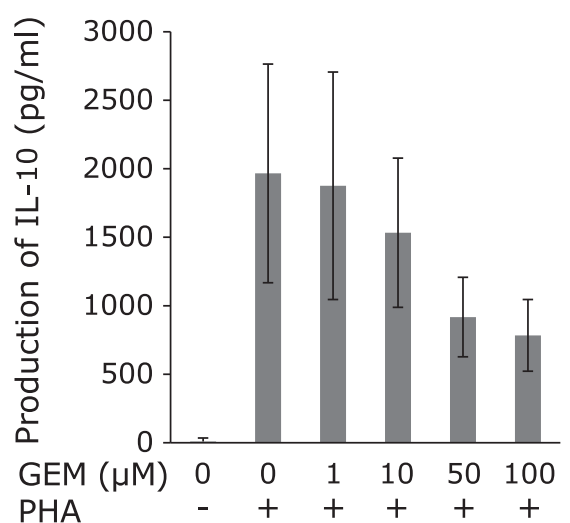

b

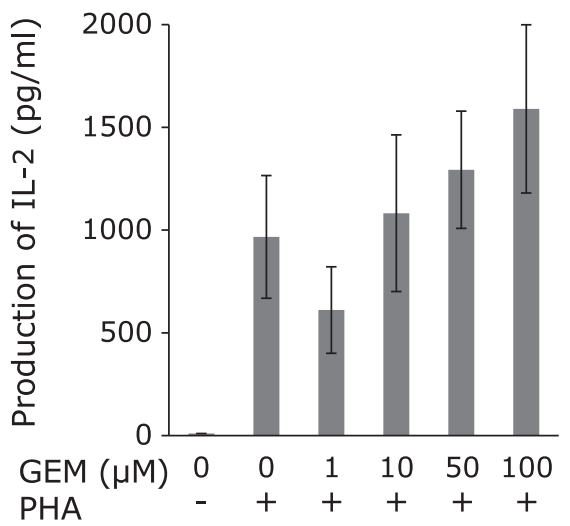

e

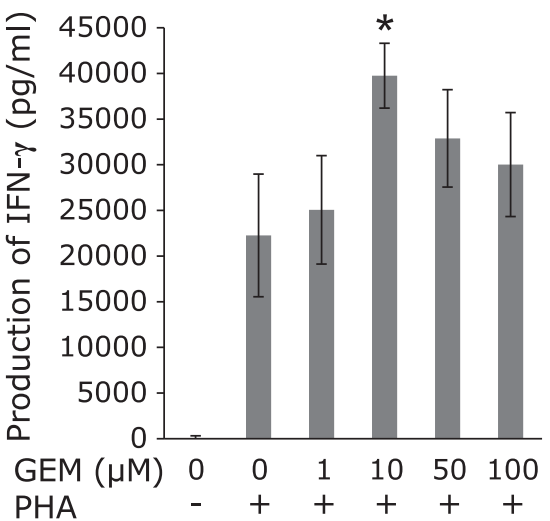

C

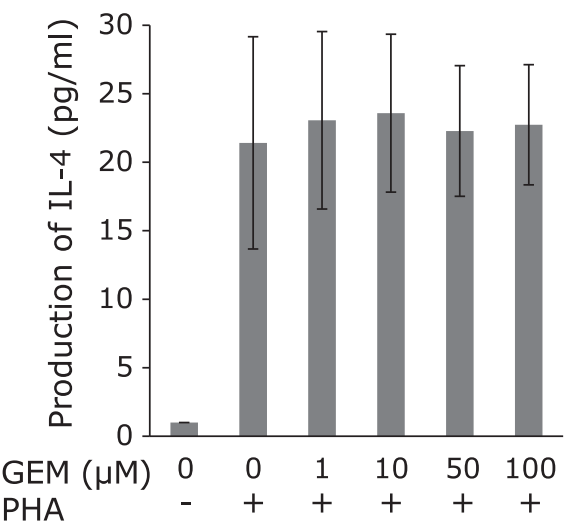

Fig. 6. Effects of GEM on PHA-induced cytokine production. After $2 \mathrm{~h}$ chemical stimulation with GEM, PBMCs were then incubated with PHA for $48 \mathrm{~h}$. Cell culture supernatant was assayed for TNF- $\alpha(a), I L-2(b), I L-4(c), I L-10(d)$ and IFN- $\gamma(e)$ by using a cytokine-specific solid phase sandwich ELISA. In all panels, data are expressed as the mean \pm SEM. ${ }^{*} p<0.05,{ }^{* *} p<0.0001$, vs PHA(+), no anticancer agent.
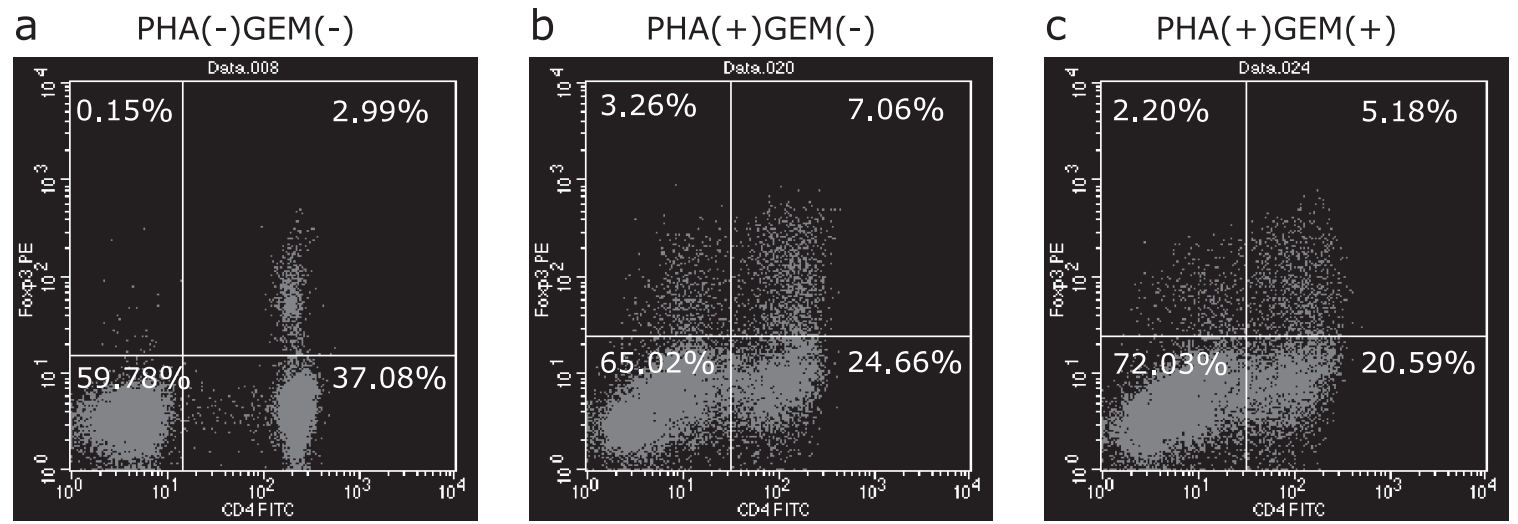

Fig. 7. A representative result of CD4 and Foxp3 dual staining and flow cytometry. After $2 \mathrm{~h}$ stimulation with GEM, PBMCs were incubated with PHA for $48 \mathrm{~h}$. CD4 and intracellular staining for Foxp3 were evaluated by flow cytometry.

findings of this study provide data that might aid in the design of combined treatment with chemotherapy and immunotherapy. Further investigation in cancer cells and clinical settings is needed to examine the potential for such combination therapy.

\section{Acknowledgments}

This study was partially supported by Grant-in-Aid for Scientific Research (C) (no. 23590891) from the Ministry of Education, Culture, Sports, Science and Technology of Japan. 

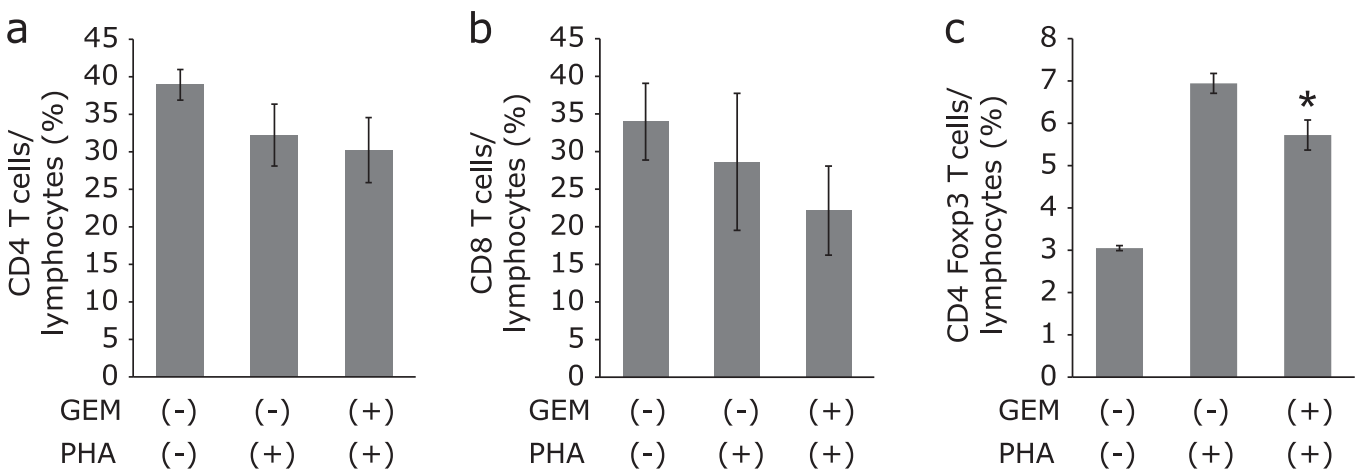

Fig. 8. Effects of GEM on lymphocyte populations. After $2 \mathrm{~h}$ stimulation with GEM, PBMCs were incubated with PHA for $48 \mathrm{~h}$. Cell types (CD4 and CD8) and intracellular staining for Foxp3 were evaluated by flow cytometry. In all panels, data are expressed as the mean \pm SEM. * $p<0.05$, vs PHA(+), GEM(-).
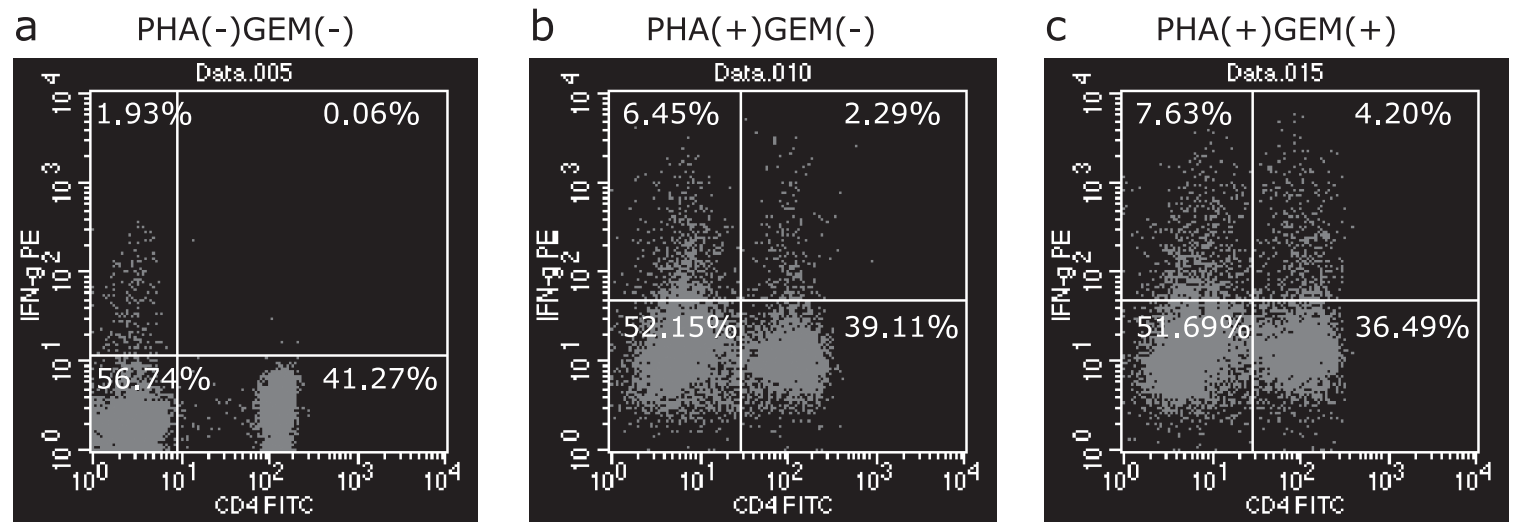

Fig. 9. A representative result of CD4 and IFN- $\gamma$ dual staining and flow cytometry. After $2 \mathrm{~h}$ stimulation with GEM, PBMCs were incubated with PHA for $48 \mathrm{~h}$. CD4 and intracellular staining for IFN- $\gamma$ were evaluated by flow cytometry.
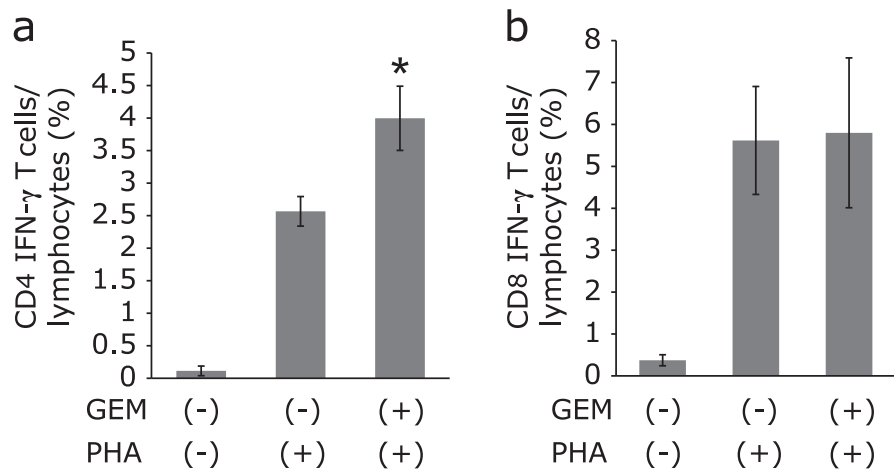

Fig. 10. Effects of GEM on the frequency of IFN- $\gamma$-producing cells. After $2 \mathrm{~h}$ stimulation with GEM, PBMCs were incubated with PHA for $48 \mathrm{~h}$ Cell types (CD4 and CD8) and intracellular staining for IFN- $\gamma$ were evaluated by flow cytometry. In all panels, data are expressed as the mean \pm SEM. * $p<0.01$, vs $\operatorname{PHA}(+)$, GEM(-).

\section{Conflict of Interest}

Satoshi Kokura, Takeshi Ishikawa and Toshikazu Yoshikawa have an affiliation with a donation-funded department from TAKARA BIO Inc. Nobuaki Yagi and Toshikazu Yoshikawa have an affiliation with a donation-funded department from
AstraZeneca Co. Ltd., Eisai Co. Ltd., Otsuka Pharmaceutical Co. Ltd., MSD K.K., Dainippon Sumitomo Pharma Co. Ltd., Chugai Pharmaceutical Co. Ltd., FUJIFILM Medical Co. Ltd. and Merck Serono Co. Ltd. Yuji Naito received research grants from Takeda Phamaceutical Co. Ltd. and Otsuka Phamaceutical Co. Ltd. The other authors have no conflict of interest to declare.

\section{References}

1 AbdAlla EE, Blair GE, Jones RA, Sue-Ling HM, Johnston D. Mechanism of synergy of levamisole and fluorouracil: induction of human leukocyte antigen class I in a colorectal cancer cell line. J Natl Cancer Inst 1995; 87: 489-496.

2 Bergmann-Leitner ES, Abrams SI. Treatment of human colon carcinoma cell lines with anti-neoplastic agents enhances their lytic sensitivity to antigenspecific $\mathrm{CD}^{+}$cytotoxic T lymphocytes. Cancer Immunol Immunother 2001 50: $445-455$.

3 Correale P, Del Vecchio MT, La Place M, et al. Chemotherapeutic drugs may be used to enhance the killing efficacy of human tumor antigen peptidespecific CTLs. J Immunother 2008; 31: 132-147.

4 Sinha P, Clements VK, Bunt SK, Albelda SM, Ostrand-Rosenberg S. Crosstalk between myeloid-derived suppressor cells and macrophages subverts tumor immunity toward a type 2 response. J Immunol 2007; 179: 977-983.

5 Hirooka Y, Ito A, Kawashima H, et al. A combination therapy of gemcitabine with immunotherapy for patients with inoperable locally advanced pancreatic cancer. Pancreas 2009; 38: e69-e74.

6 Suzuki E, Sun J, Kapoor V, Jassar AS, Albelda SM. Gemcitabine has significant immunomodulatory activity in murine tumor models independent of its cytotoxic effects. Cancer Biol Ther 2007; 6: 880-885.

7 Prete SP, Turriziani M, Massara MC, et al. Combined effects of 5-fluorouracil, 
folinic acid and oxaliplatin on the expression of carcinoembryonic antigen in human colon cancer cells: pharmacological basis to develop an active antitumor immunochemotherapy. J Exp Clin Cancer Res 2008; 27: 5.

8 Zitvogel L, Apetoh L, Ghiringhelli F, Kroemer G. Immunological aspects of cancer chemotherapy. Nat Rev Immunol 2008; 8(1): 59-73.

9 Correale P, Cusi MG, Micheli L, et al. Chemo-immunotherapy of colorectal carcinoma: preclinical rationale and clinical experience. Invest New Drugs 2006; 24: 99-110.

10 Alvino E, Fuggetta MP, Tricarico M, Bonmassar E. 2'-2'-difluorodeoxycytidine: in vitro effects on cell-mediated immune response. Anticancer Res 1998; 18: 3597-3602.

11 Tiefenthaler M, Hohla F, Irschick E, et al. In vitro studies on the immunosuppressive effect of 2',2'-difluorodeoxycytidine $(\mathrm{dFdC})$ and its metabolite 2',2'-difluorodeoxyuridine (dFdU). Immunobiology 2003; 207: 149-157.

12 Margreiter R, Fischer M, Roberts K, et al. Gemcitabine-a novel immunosuppressive agent-prevents rejection in a rat cardiac transplantation model. Transplantation 1999; 68: 1051-1053.

13 Nowak AK, Robinson BW, Lake RA. Gemcitabine exerts a selective effects on the humoral immune response: implications for combination chemoimmunothrapy. Cancer Res 2002; 62: 2353-2358.

14 Agarwal A, Verma S, Burra U, Murthy NS, Mohanty NK, Sexana S. Flow cytometric analysis of Th1 and Th2 cytokines in PBMCs as a parameter of immunological dysfunction in patients of superficial transitional cell carcinoma of bladder. Cancer Immunol Immunother 2006; 55: 734-743.

15 Hadden JW. Recent advances in the preclinical and clinical immunopharmacology of interleukin-2: emphasis on IL-2 as an immunorestrative agent. Cancer Detect Prev 1988; 12: 537-552.

16 Le HK, Graham L, Cha E, Morales JK, Manjili MH, Bear HD. Gemcitabine directly inhibits myeloid derived suppressor cells in BALB/c mice bearing 4T1 mammary carcinoma and augments expansion of $\mathrm{T}$ cells from tumor- bearing mice. Int Immunopharmacol 2009; 9: 900-909.

17 Plate JM, Plate AE, Shott S, Bograd S, Harris JE. Effects of gemcitabine on immune cells in subjects with adenocarcinoma of the pancreas. Cancer Immunol Immunother 2005; 54: 915-925.

18 Suzuki E, Kapoor V, Jassar AS, Kaiser LR, Albelda SM. Gemcitabine selectively eliminates splenic Gr-1+/CD11b+ myeloid suppressor cells in tumor-bearing animals and enhances antitumor immune activity. Clin Cancer Res 2005; 11: 6713-6721.

19 de Rezende, Silva IV, Rangel LB, Guimaraes MC. Regulatory T cells as a target for cancer therapy. Arch Immunol Ther Exp (Warsz) 2010; 58: 179190.

20 Nicholl M, Lodge A, Brown I, Sugg SL, Shilyansky J. Restored immune response to an MHC-II-Restricted antigen in tumor-bearing hosts after elimination of regulatory T cells. J Pediatr Surg 2004; 39: 941-946.

21 Barnett B, Kryczek I, Cheng P, Zou W, Curiel TJ. Regulatory T cells in ovarian cancer: biology and therapeutic potential. Am J Reprod Immunol 2005; 54: 369-377.

22 Dannull J, Su Z, Rizzieri D, et al. Enhancement of vaccine-mediated antitumor immunity in cancer patients after depletion of regulatory T cells. J Clin Invest 2005 ; 115: 3623-3633.

23 Shimizu J, Yamazaki S, Sakaguchi S. Induction of tumor immunity by removing $\mathrm{CD} 25+\mathrm{CD} 4+\mathrm{T}$ cells: a common basis between tumor immunity and autoimmunity. J Immunol 1999; 163: 5211-5218.

24 Onizuka S, Tawara I, Shimizu J, Sakaguchi S, Fujita T, Nakayama E. Tumor rejection by in vivo administration of anti-CD25 (interleukin-2 receptor alpha) monoclonal antibody. Cancer Res 1999; 59: 3128-3133.

25 Kimura Y, Tsukada J, Tomoda T, et al. Clinical and immunologic evaluation of dendritic cell-based immunotherapy in combination with gemcitabine and/or S-1 in patients with advanced pancreatic carcinoma. Pancreas 2012; 41: $195-205$. 